Distintos principios, consecuencias enfrentadas: la oposición entre la consideración moral de los animales y el ecologismo

Oscar Horta

Universidad de Santiago de Compostela

Oscar.Horta@usc.es

\title{
Introducción
}

El ámbito de estudio conocido como ética animal examina cuál es la consideración moral que deberíamos dar a los animales no humanos y cuáles son las consecuencias prácticas. Dentro de este campo destacan las posiciones que defienden que todos los seres sintientes deben ser objeto de respeto al margen de su especie.

En contraste, la ética medioambiental examina si deberíamos considerar a entidades como ecosistemas, biocenosis, paisajes o especies, y qué implicaciones tendría. En este campo han destacado distintas posiciones. Dos ejemplos son el biocentrismo y el ecocentrismo, que defienden, respectivamente, la consideración moral de todas las entidades vivas y de los ecosistemas en tanto que conjuntos. 
Estas posiciones, al diferir acerca de las razones por las que debemos dar respeto a alguien o a algo, tienen corolarios prácticos muy dispares, y a menudo opuestos ${ }^{1}$. Veremos a continuación en qué consisten los conflictos que, así, pueden surgir entre ellas. Al hacerlo, examinaremos también hasta qué punto es plausible cada una de estas posiciones.

\section{El biocentrismo}

El biocentrismo es la posición que estima que las entidades moralmente considerables son los seres vivos. Asume, pues, que el cumplimiento de las funciones vitales constituyen la condición suficiente y necesaria para tal consideración, o bien, garantiza la satisfacción de tal condición si ésta es distinta del propio cumplimiento de esas funciones. Sería así independientemente de cuáles sean otras capacidades que posean los seres dotados de ella. Se ha considerado comúnmente a Albert Schweitzer como el precursor de esta posición (Schweitzer, 1962). Asimismo, se han identificado con ella: Kenneth Goodpaster (1978: 108-120), Paul Taylor (1986) o Gary Varner (2002), quienes han afirmado que la razón para tenerlos en cuenta radicaría en que todos los seres vivos pueden ser perjudicados o beneficiados: a estos seres las cosas les pueden ir bien o mal (Taylor, 1986: 75)².

1 Un estudio sobre la bibliografía que trata estas cuestiones se puede encontrar en Dorado (2015).

2 Esta misma idea es defendida en Attfield, 1987: 47-58. 


\section{Por qué es cuestionable el biocentrismo}

Hay motivos para cuestionar la pertinencia del criterio biocéntrico. Considérese el siguiente experimento mental. Supongamos que caemos en las manos de unos torturadores que nos comunican que pasaremos el resto de nuestra vida padeciendo los más terribles suplicios y sin posibilidad alguna de escape. Ante esta perspectiva, la mayoría pensaremos que lo mejor sería morir. Lo que esto nos muestra es que el motivo por el que valoramos la vida radica en las experiencias que podemos vivir en ella, no en el mero hecho de estar vivos. De hecho, cuando hablamos de beneficiar o dañar a un ser vivo no consciente estamos dando a estos términos un sentido diferente al que empleamos cuando hablamos de bienestar experiencial, es decir, entendido en términos de lo positivas o negativas que sean las experiencias que poseemos. Este sentido choca con lo que a la mayoría nos parece más intuitivo. En nuestra vida valoramos tales bienes en la medida en que nos llevan a poder disfrutar de beneficios experienciales, pero no por sí mismos. Por ejemplo: mantener un corazón saludable es positivo porque nos permite disfrutar de la vida. Sin embargo, normalmente consideramos que si entramos en un coma irreversible la salud de nuestro corazón deja ya de resultar algo valioso. Es así porque una vida sin experiencias de ningún tipo nos parece un vacío indistinguible de la no existencia, asumiendo una teoría de la identidad que nos reconoce como organismos vivos, aunque otras defienden que realmente no somos organismos vivos. Según estas teorías, lo que somos es algo distinto, como conjuntos de estados mentales, cerebros, la actividad de nuestros sistemas nerviosos, etc. Estas entidades serían, conforme a estas creencias, lo moralmente considerable, y no las funciones vi- 
tales que garantizarían su existencia. En cualquier caso, al margen de cuál sea nuestra teoría de la identidad, tenemos razones para entender que el motivo por el que contamos con intereses moralmente importantes es que podemos vivir experiencias positivas y negativas.

Así, encontramos que una vida sin experiencias (como sería, por ejemplo, la de una bacteria o una planta, o la de un organismo animal en estado vegetativo permanente) es un vacío en el que no hay presente nada de aquéllo que resulta valioso. Si entendemos que la consideración moral debería depender de aquello que resulta valioso, tendríamos que concluir que el mero hecho de estar vivo no es lo que hace que alguien deba ser moralmente considerable.

Por otra parte, el biocentrismo conduce al surgimiento de conflictos de intereses muy serios y de difícil solución y ocurre en todos los casos en los que la posibilidad de que unos seres vivan, impide que otros puedan hacerlo. El respeto por todas las formas de vida llevaría a adoptar soluciones inciertas, y que para quienes asuman otros criterios (como la necesidad de respetar a aquellos seres con la capacidad de sufrir y disfrutar) resultarán inaceptables. Pensemos en lo que ocurriría en el caso del choque de intereses, presente de forma continua, entre los microorganismos y otras formas de vida dotadas de la posibilidad de experimentar bienestar. Una posición biocéntrica nos llevaría a inclinar la balanza hacia un imperio de bacterias y otros microorganismos, en perjuicio de otras formas de vida. Como también ha señalado Peter Singer, de ser así, el abnegado trabajo de Schweitzer -como doctor en África- resultaría objetable por ocasionar la muerte de innumerables gérmenes (Singer, 1979: 200). Debemos tener presente que si el criterio por el que nos debemos guiar para considerar moralmente a una entidad es que 
esté viva, entonces en casos de conflicto careceremos de una pauta para dar prioridad a unos seres por encima de otros. Si optamos por salvar la vida del animal es porque nos estamos guiando por otros principios distintos al que prescribe el respeto por la vida. De ese modo, hablando en rigor, lo que hacemos es adoptar un criterio diferente. Pasamos a sostener una posición de carácter pluralista ${ }^{3}$. Esto es, combinamos el criterio ligado a la posesión de una vida con otro ligado a la sintiencia, pero sucede que ésta podría ser también satisfecha por alguna entidad no viva. Así, una inteligencia artificial futura que fuese sintiente tendría que ser moralmente considerable, aunque no estuviese viva. Sin embargo, esa posición ya no sería biocéntrica. La única posición pluralista compatible con el biocentrismo sería alguna que manejase un criterio para la consideración moral que cubriese a los seres vivos y sólo a ellos, y otro que únicamente fuese satisfecho por algunos seres vivos, y que no lo fuese por ninguna entidad no viva. Las capacidades cognitivas complejas pueden satisfacer este requisito al día de hoy, pero en el futuro es posible (de hecho, es muy probable) que ya no sea así, en el momento en el que haya inteligencias artificiales que las posean ${ }^{4}$.

Hay además otro corolario del biocentrismo que resultará difícilmente admisible: el hecho que niega la posesión de nuestra propia vida. Si una vida es algo valioso por sí misma, independientemente de la capacidad de disfrute o sufrimien-

3 Una posible respuesta pasaría por afirmar que el cuerpo del animal enfermo estaría a su vez compuesto por células vivas que habría igualmente que respetar. Con todo, ello sigue chocando con lo que la mayoría pensamos, pues lo que normalmente valoramos del hecho de vivir no es la vida de las células que componen nuestro organismo.

4 Esto es, no inteligencias artificiales como las que existen hoy en día, sino inteligencias artificiales generales que puedan solucionar problemas en un amplio rango, como los que pueden solucionar hoy nuestros cerebros. Ver sobre esto Mannino, A., et al. (2015); Tomasik, B. (2016). 
to de quien la vive, entonces su valor es independiente de nuestra opinión como seres que la vivimos. El suicidio deberá ser condenado por suponer una forma de asesinato. Igualmente será censurable toda práctica que implique un daño o peligro para nuestra vida (desde el consumo de comida poco saludable hasta el deporte de riesgo). Esta conclusión es difícil de aceptar, ya que implica una desconsideración completa por la satisfacción de nuestros intereses.

\section{Subordinación del biocentrismo al antropocentrismo}

Quienes en principio han simpatizado con el biocentrismo optaron por la combinación de un criterio ligado a la posesión de una vida con posiciones antropocéntricas. El antropocentrismo es la idea de que la satisfacción de los intereses humanos es lo central para la moral ${ }^{5}$. Lo que no implica necesariamente que los únicos intereses a considerar sean los humanos. Simplemente supone que en caso de conflicto entre intereses humanos e intereses de otros seres, la satisfacción de los primeros debe resultar prioritaria. Así, la combinación de biocentrismo con el antropocentrismo presupone que además de dar consideración a todos los seres vivos, se brinda una atención especial a los seres humanos.

Hay distintas formas en las que esa combinación podría llevarse a cabo. La que prevalece entre quienes defienden el biocentrismo es la que da predominancia al antropocentris$\mathrm{mo}^{6}$. Lo que esto implica es que se prescribe respeto a los

5 Se está hablando aquí de antropocentrismo en sentido moral, y no, por ejemplo, de un antropocentrismo epistémico que plantease que nuestro punto de vista humano determina nuestra forma de ver las cosas, aun cuando éste pueda ser también cuestionado.

6 Pueden consultarse distintos ejemplos de este posicionamiento en Attfield, 1987; Wenz, 1988: 291; Varner 2002: 79; Schweitzer 1962: 354. 
seres vivos, salvo cuando haya un interés humano mínimamente significativo que se vea frustrado al hacerlo.

Al respecto, es preciso hacer un apunte de cuestiones terminológicas cuya importancia no debería desdeñarse. Se ha distinguido, en ocasiones, entre biocentrismo «fuerte»y «débil» ${ }^{7}$. Esta terminología lleva a la confusión, pues lo que ocurre no es que se asuman dos criterios distintos en cada caso, o que el adquirido permita distintos grados en su aplicación. Lo que sucede es que, por una parte, puede defenderse un enfoque biocéntrico y, por otra, una combinación del biocentrismo con otros criterios.

\title{
El ecocentrismo
}

Se llama ecocentrismo a la posición que sostiene que las entidades moralmente valiosas no son los individuos, sino los ecosistemas en los que viven. El autor que comúnmente ha sido identificado con este enfoque es John Baird Callicott (1989). Como precedente de su posición se ha apuntado, a su vez, a Aldo Leopold, quien dictaría el que se convirtió en el pasaje más frecuentemente identificado con el ecocentrismo:

\begin{abstract}
Algo es correcto cuando tiende a preservar la integridad, estabilidad y belleza de la comunidad biótica. Es incorrecto cuando tiende a otra cosa (Leopold, 2000: 155).
\end{abstract}

7 Ver, por ejemplo, Einar Himma, 2004: 25-44. Siguiendo esta terminología (muy objetable) el «biocentrismo fuerte» sostendría que la posesión de una vida es el único criterio a satisfacer para ser considerable moralmente. A su vez, el «débil» indicaría que el hecho de estar vivo resultaría relevante, pero no lo único, de manera que constituiría un factor a considerar junto a otros posibles criterios también moralmente relevantes. 
El ecocentrismo es un tipo de posición holista, donde las entidades moralmente considerables son conjuntos $^{8}$. Una forma equivocada de entenderla es por creer que lo que el ecocentrismo defiende como relevante a la hora de conservar un sistema natural es la defensa de los individuos que en él habitan. Pensaríamos que lo negativo de dañar a una comunidad o lo positivo de beneficiarla radicaría en el beneficio o perjuicio que se ocasionaría a los individuos que la componen. Esta visión, sin embargo, no se corresponde con la que en realidad sostiene una posición holista como el ecocentrismo y que valora las comunidades en cuanto tales.

\section{El cuestionamiento del ecocentrismo}

Acabamos de constatar así la recursividad implicada en las defensas del ecocentrismo y que afirman que la no consideración de los ecosistemas perjudica los individuos que habitan en ellos. Para el ecocentrismo, lo negativo no será, en última instancia, el daño que sufran sus miembros, sino el padecido por la comunidad en la que recae realmente la posesión de valor. Si mantenemos que lo objetable de la destrucción de las comunidades bióticas es el daño que sufren sus miembros, estamos abandonando la perspectiva ecocéntrica, y sosteniendo que las comunidades poseen valor instrumental para los individuos. Esto parece difícil de aceptar. Observamos normalmente que son los individuos, y no los conjuntos, quienes son moralmente considerables. Mucha gente puede pensar que la continuidad de ciertos conjuntos puede ser positiva, pero porque lo es para los individuos, no por ella sola.

8 Otros ejemplos de posiciones holistas distintas del ecocentrismo serían aquéllas que dan valor a la conservación de las especies en lugar de a los miembros de éstas. 
Las consecuencias del ecocentrismo serían, al igual que las del biocentrismo, difíciles de aceptar. Así, cuando el bien de un ecosistema lo requiera, desde esta posición tendremos que bendecir el sacrificio en masa de sus componentes (entre quienes, obviamente, nos encontraremos también los seres humanos). El motivo para proceder así no es que se busque el bien de todos los demás individuos, como se ha indicado ya, sino el bien de la comunidad. Más adelante vamos a ver algunos ejemplos del modo en el que se está presentado en la actualidad.

\section{Subordinación del ecocentrismo al antropocentrismo}

Quienes se han alineado con el ecocentrismo mantienen, al llegar a este punto, que, en la medida en que los seres humanos se ven afectados, otros factores han de tener prioridad por encima de la defensa de los ecosistemas. De tal forma, sucederá algo semejante a lo que ocurría en el caso del biocentrismo. La postura resultante será una combinación de distintas condiciones, que en ciertos momentos tenga en cuenta un criterio ecocéntrico que se abandone más tarde, cuando el otro principio, de carácter antropocéntrico, entre en juego (Varner, 1991: 175-179). Ya Leopold habría sostenido esta opinión (2000: 135). Nuevamente, el autor cuyo punto de vista puede catalogarse de forma más representativa con esta combinación entre ecocentrismo y antropocentrismo es Callicott (1990: 99-124; 2000: 204-217). Él considera que nuestras obligaciones morales han de dirigirse a aquellas entidades situadas dentro de una serie de círculos concéntricos. En los más cercanos se encontrarían aquellos seres humanos con los que tenemos relaciones más próximas, y en los más lejanos las demás entidades moralmente considerables. Y para solventar los posibles conflic- 
tos que puedan surgir entre los intereses de los receptores de consideración moral situados en distintos círculos, dicta dos principios de segundo orden. El primero sostendría que los intereses de quienes se encontrasen en los círculos más cercanos tendrían prioridad (Callicott, 2000: 211). Y el segundo, que los intereses más importantes tienen mayor peso que los menos importantes (Callicott, 2000: 211).

Aunque Leopold y Callicott han sido considerados de forma general como los principales valedores del ecocentrismo, en realidad el enfoque que asumen es ecocéntrico de manera subordinada a los intereses humanos ${ }^{9}$.

\section{El amplio apoyo disfrutado por el antropocentrismo}

Los planteamientos antropocéntricos han sido asumidos igualmente por un gran número de autores no alineados con el biocentrismo o el ecocentrismo, entre quienes han destacado en la llamada ecología profunda están: Arne Næss, 1989; Bill Devall, George Sessions, 1985 o Warwick Fox, 1995. La idea que implica la adopción de un punto de vista no antropocéntrico está muy extendida, pero es errónea. La ecología profunda reconoce consideración moral a entidades no humanas. Lo anterior supone rechazar la parcialidad a favor de los seres humanos ${ }^{10}$. Podemos considerar que tanto los humanos como los demás seres sintientes merecen respeto, pero se da un respeto extra a los primeros, aun en los casos en los que sus intereses no son más importantes. Y, de hecho,

9 En el caso de Callicott esto resulta chocante, dada su fuerte oposición al pluralismo.

10 La ecología profunda se define por la adscripción a la llamada "plataforma de los ocho puntos». En ésta, como se ha indicado, se defiende el valor intrínseco de los seres humanos y no humanos que forman parte de la biosfera, pero no su consideración igualitaria. Ver Næss \& Sessions, 1999: 8-9. 
es lo que han propuesto los ecologistas profundos citados arriba.

En el caso de la denominada ecología social, su representante más conocido, Murray Bookchin (1994), ha destacado por su ataque explícito al antiantropocentrismo. A su vez, dentro del ecofeminismo encontramos posiciones de lo más diversas, pudiéndose distinguir las siguientes: (i) aquéllas centradas fundamentalmente en los intereses humanos de conservar ciertos ecosistemas (como las de Vandana Shiva, 1992 o Barbara Hollad-Cunz, 1996); (ii) aquéllas que tematizan la cuestión del antropocentrismo, pero sin rechazarlo (como las de Karen Warren, 2000 o Val Plumwood, 1993); y (iii) aquéllas que efectivamente cuestionan el antropocentrismo (como las de Carol J. Adams, 1994 o Josephine Donovan, 2006: 305-29). Se trata, pues, de una perspectiva con representantes no antropocentristas, que constituyen una minoría. Por último, habrá también otras voces destacadas dentro de la ética medioambiental no posicionadas en ninguna de las categorías anteriores, como es el caso de Eugene Hargrove, Bryan Norton o Mary Anne Warren (Hargrove,1992), quienes han asumido la parcialidad en contra de los seres no humanos.

Vemos así que se debe dejar a un lado la idea de que la ética medioambiental actual rompe con los planteamientos antropocéntricos tradicionales. La mayor parte de sus representantes sólo los cuestiona de una forma muy limitada y, en muchos casos, basan en ellos la recomendación de que el medio ambiente sea respetado. Es normal que así sea, ya que hay un gran número de intereses humanos implicados: económicos, científicos, médicos, recreacionales, estéticos, emocionales. De hecho, lo que en muchos casos se da no es un conflicto entre los intereses humanos y los de los individuos no humanos, o entre intereses humanos y la conservación 
de un ecosistema, sino entre algunos intereses humanos por conservar el medio y otros que implican su destrucción.

\section{La crítica al antropocentrismo}

Pese al amplio apoyo que, como vemos, tienen las posiciones antropocéntricas, algunos de los argumentos apuntados en los apartados anteriores llevan a dudar seriamente de su validez. Al cuestionar las posiciones biocéntricas y ecocéntricas vimos razones para concluir que el requisito para la consideración moral debería radicar en la posibilidad de poseer experiencias. Ahora bien, si esto es lo que hace que un ente deba ser considerado moralmente, entonces todos aquéllos seres que puedan tener experiencias positivas y negativas habrán de ser considerados, y no únicamente aquellos que pertenezcan a la especie humana. La simple pertenencia a una especie es algo no relacionado con la capacidad que alguien tiene para vivir experiencias positivas y negativas. Esto nos da razones para rechazar que se preste atención preferente a los seres humanos, ya que se puede afirmar que tal posición constituye una forma de especismo: una discriminación de quienes no pertenecen a una cierta especie (Ryder, 2010: 1-2; Horta, 2008; Riechmann: 107-118; Rachels, 1990; Pluhar, 1995; Cavalieri, 2001; Bernstein, 2015; Dorado, 2010: 47-63).

Si los argumentos son correctos, podremos decir que el criterio que defina lo que resulte moralmente relevante será uno distinto al antropocéntrico y a los propios de posturas biocentristas y ecocentristas. Consistirá en la capacidad de tener experiencias poseídas por un gran número de animales no humanos, pero no por los seres vivos que no poseen sistemas nerviosos centralizados, ni tampoco por entidades como los ecosistemas. 
Observamos las claras diferencias que existen entre las posiciones centradas en la sintiencia, que defiende la consideración moral de cualquier ser que pueda sufrir y disfrutar, y las que defienden las posiciones ecologistas. Veremos a continuación las consecuencias prácticas que se siguen.

La oposición entre la consideración de los animales y el ecologismo, en la práctica

Los conflictos prácticos más concretos que se presentan entre los distintos puntos de vista revisados tienen lugar fundamentalmente en dos ámbitos:

(i) El primero es el relativo a la explotación animal. La posición centrada en la sintiencia rechazará aquélla, dados los graves daños que inflige a los animales que la sufren. A su vez, las posiciones ecologistas se opondrán a tal explotación si ésta tiene un impacto negativo en la conservación medioambiental, pero la aceptarán en caso contrario.

(ii) El segundo consiste en la actitud hacia los animales que habitan en la naturaleza, y en concreto hacia la clase de intervenciones con las que podemos afectarlos para bien y para mal. Si observamos que los entes moralmente considerables son los seres sintientes, rechazaremos aquellas intervenciones en los procesos naturales que dañen a los animales con el fin de, por ejemplo, mantener una cierta especie, o conservar o modelar un determinado ecosistema. Son numerososos los casos en los que sucede. En ocasiones se emprenden matanzas de animales para evitar que se reproduzcan con otras especies cuyos rasgos distintivos se busca conservar. Un ejemplo de lo anterior fue el exterminio de malvasías 
canelas para evitar su hibridación con las malvasías cabeciblancas del sur de Europa. En otros casos se realizan matanzas de animales herbívoros que amenazan la existencia de algunas especies de plantas, como ha sucedido en algunas islas californianas (Rolston, 1999; Shelton, 2004: 1-21; Mosquera, 2015: 65-77). Otro ejemplo de intervención negativa para los animales lo encontramos en la introducción de depredadores para mantener bajo control a una población de potenciales presas y que se lleva a cabo en áreas donde se busca prevenir el crecimiento de las poblaciones de herbívoros, o preservar las plantas con las que éstos se alimentan. La medida es negativa para los depredadores afectados (que son capturados, separados de sus familias y llevados a un entorno desconocido); pero, como es obvio, lo es sobre todo para sus presas, que mueren y sufren por el miedo y por otras limitaciones que se les imponen (Horta, 2015: 61-85).

\section{Intervenciones en la naturaleza favorables a los animales}

Otras contraposiciones entre la defensa de los animales y el ecologismo surgen cuando consideramos intervenciones que no son dañinas, sino positivas para los animales. Contra lo que se piensa en muchas ocasiones, las vidas de los animales en la naturaleza no son idílicas; al contrario, suelen ser cortas y llenas de sufrimiento. Los animales salvajes padecen daños notables de forma cotidiana como resultado de los procesos naturales. Sufren continuamente por causas como el hambre y la sed, enfermedades y lesiones, condiciones climáticas hostiles, ataques de otros animales y parasitis- 
mo, estrés psicológico, etcétera ${ }^{11}$. Prácticamente, la totalidad de los animales muere poco después de nacer. La estrategia reproductiva que prevalece masivamente en la naturaleza consiste en traer al mundo a gran número de animales. Algunos roedores pueden tener más de cien crías a lo largo de su vida, muchos anfibios pueden depositar miles de huevos en cada puesta, mientras que los peces lo hacen con millones cada vez. De todos los animales sintientes que nacen de sus huevos, en poblaciones más o menos estables, sólo sobrevive en promedio uno por cada hembra; la inmensa mayoría muere, normalmente, poco después de comenzar a existir, y por lo habitual de forma dolorosa. Al ser tan jóvenes, no tienen tiempo de disfrutar de experiencias positivas. Así, hay razones de mucho peso para concluir que la mayoría de los animales que vienen al mundo tienen más sufrimiento que disfrute en sus vidas ${ }^{12}$.

Por estos motivos, si hay formas de intervenir para reducir tales daños, las posturas a favor de los animales defenderán que se lleven a cabo, incluso aunque ello suponga alterar los procesos naturales. Lo que nos debe importar no son las relaciones ecosistémicas, sino los intereses de los seres sintientes afectados por éstas. En cambio, desde las posiciones ecologistas tales intervenciones serán rechazadas, pues, conforme a éstas, los procesos naturales son positivos aunque tengan un impacto negativo a título individual en los animales ${ }^{13}$.

11 Ver sobre esto Ética animal, 2016a o, en lo que toca a aspectos más concretos de los daños naturales que sufren los animales no humanos, Wobeser, 2005; Zimmerman 2009: 1-7; Bunke et al., 2015.

12 Ver Tomasik, 2010: 133-152; Horta, 2011: 57-75; Dorado, 2015: 37-42; Faria, 2016.

13 La posición ecologista contraria a intervenir a favor de los seres sintientes se defiende por ejemplo en Callicott, 1980: 311-338; Sagoff, 1984: 297-307 o Rolston III, 1992: 250-278. La posición favorable a ayudar a los animales y en contra de las objeciones ecologistas 
Por qué el biocentrismo no defiende necesariamente posiciones ecologistas

Las posiciones que se oponen a la intervención en el mundo natural con el fin de ayudar a individuos concretos, han de ser necesariamente de carácter holista, como el ecocentrismo. En cambio, el biocentrismo tendría que reconocer que si para la mayor parte de las entidades individuales que se encuentran en la naturaleza hay más desvalor que valor positivo, será una situación que se evaluaría en conjunto como negativa. En realidad, no todas las posiciones éticas que se han asumido y que se encuentran ligadas al ecologismo se tendrían que oponer a la intervención de la naturaleza a favor de los animales. Esto puede incluso cuestionar que el biocentrismo sea propiamente una posición ecologista, aunque siga estando en conflicto en otra serie de puntos con la posición centrada en la consideración de los seres sintientes.

\section{Conclusión}

Lo que hemos visto en los anteriores apartados muestra que es incorrecta la idea de que la consideración de los animales está relacionada con el ecologismo. De hecho, se dan conflictos patentes entre ambas posturas. Las bases éticas de las que parten son claramente distintas, y lo son también las consecuencias que prescriben. Se espera que esta oposición se ponga de manifiesto de forma más acentuada en el futuro, conforme aumente la consideración por los animales no hu-

ha sido defendida, por ejemplo, en Nussbaum, 2006; Torres Aldave, 2015: 39-53; Faria, 2016; Ética animal, 2016b. 
manos y se conozca más la situación de gran necesidad en la que se encuentran éstos en la naturaleza.

Incluso, hemos visto que entre las posiciones tradicionalmente defendidas como ecologistas hay serias divergencias. Algunas de ellas, en concreto las biocentristas, podrán aliarse con posiciones holistas como el ecocentrismo en ciertas ocasiones. No será así en el caso de la intervención en la naturaleza en beneficio de los seres sintientes. En función de las circunstancias, el biocentrismo podrá estar a favor de ésta, en contra de la postura no intervencionista defendida por posturas ecologistas como el ecocentrismo.

La idea de que deberíamos ayudar a los animales en situación de necesidad, aunque ello suponga realizar transformaciones importantes en los procesos naturales, puede resultar contraintuitiva. Como hemos visto, tenemos razones de peso para defenderla, como también lo haríamos cuando quienes necesitan la ayuda son los seres humanos.

\section{Bibliografía}

Adams, C. (1994). Neither Man Nor Beast: Feminism and the Defence of Animals. New York: Continuum; Donovan, J. (2006). Feminism and the Treatment of Animals: From Care to Dialogue. Signs, 31.

Attfield, R. (1987). Biocentrism, Moral Standing and Moral Significance. Philosophica, 39.

Bernstein, M. H. (2015) The Moral Equality of Humans and Animals. Basingstone: Palgrave Macmillan.

Bookchin, M. (1994) Which Way for the Ecology Movement. San Francisco: AK Press. 
Bunke, M., Alexander, M. E., Dick, J. T. A., Hatcher, M. J., Paterson, R. \& Dunn, A. M. (2015). Eaten alive: Cannibalism is enhanced by parasites. Royal Society Open Science, 2, 140369.

Callicot, J.B. (2000). The Land Ethic. En Jamieson, D. (ed.) A Companion to Environmental Philosophy. Oxford: Blackwell. Callicott, J. B. (1989). In Defense of the Land Ethic: Essays in Environmental Philosophy. Albany: SUNY Press.

Callicott, J. B. (1990). The Case against Moral Pluralism. Environmental Ethics, 12, 99-124; y (2000) The Land Ethic. En Jamieson, D. (ed.) A Companion to Environmental Philosophy. Oxford: Blackwell.

Callicott, J. B. (1980). Animal Liberation: A Triangular Affair. Environmental Ethics, 2.

Cavalieri, P. (2001). The Animal Question: Why Nonhuman Animals Deserve Human Rights. Oxford: Oxford University Press.

Devall, B. \& Sessions, G. (1985). Deep Ecology: Living as if Nature Mattered, Salt Lake City: Gibbs Smith.

Dorado, D. (2015). El conflicto entre la ética animal y la ética ambiental: bibliografía analitica. Tesis doctoral, Madrid: Universidad Carlos III.

Dorado, D. (2015). Una aproximación bibliográfica a la cuestión de la intervención en la naturaleza. En VV.AA, Los retos de la Filosofía en el siglo XXI: Actas del I Congreso Internacional de la Red Española de Filosofía, 17, Valencia: Universitat de València.

Dorado, D. (2010). La consideración moral de los animales no humanos en los últimos cuarenta años: una bibliografía anotada. Télos, 17, http: / / www.usc.es / revistas / index.php/telos/article/view/282/248 [visitado el 27 de noviembre de 2016]. 
Einar Himma, K. (2004). Moral Biocentrism and the Adaptive Value of Consciousness. Southern Journal of Philosophy, 42.

Ética animal. (2016a). La situación de los animales en el mundo salvaje. Animales en la naturaleza, ética animal, http: / / www.animal-ethics.org/la-situacion-de-los-animalesen-el-mundo-salvaje [visitado el 23 de enero de 2017].

Ética animal. (2016b). «Ayudando a los animales en la naturaleza», Animales en la naturaleza, ética animal, http:/ / www.animal-ethics.org/ayudando-a-los-animales-enla-naturaleza [visitado el 23 de enero de 2017].

Faria, C. (2016). Animal Ethics Goes Wild: The Problem of Wild Animal Suffering and Intervention in Nature. Tesis doctoral, Barcelona: Universitat Pompeu Fabra.

Fox, W. (1995) Toward a Transpersonal Ecology: Developing New Foundations for Environmentalism, Albany: SUNY Press. González M. I., Riechmann, J., Jiménez Carreño, J. \& Tafalla, M. (coords.) Razonar y actuar en defensa de los animales. Madrid: Los libros de la catarata.

Goodpaster, K. (1978) On Being Morally Considerable. Journal of Philosophy, 75.

Hargrove, E. (1992) Foundations of Wildlife Protection Attitudes. En Hargrove, E. (ed.) The Animal Rights/Environmental Ethics Debate: The Environmental Perspective. Albany: SUNY Press.

Holland-Cunz, B. (1996 [1994]) Ecofeminismos. Madrid: Cátedra [título original: Soziales Subjekt Natur. Natur- und Geschlechterverhältnis in emanzipatorischen politischen Theorien. Frankfurt am Main: Campus].

Horta, O. (2015). Contra la ética de la ecología del miedo: por un cambio en los fines de la intervención en la naturaleza. Revista Latinoamericana de Estudios Críticos Animales, 3. 
. (2011). La cuestión del mal natural: bases evolutivas de la prevalencia del desvalor. Ágora: Papeles de Filosofía, 30.

. (2008). Términos básicos para el análisis del especismo.

Leopold, A. (2000 [1949]). Una ética de la tierra. Madrid: Los libros de la catarata, p. 155 [título original: A Sand County Almanac. Oxford: Oxford University Press].

Mannino, A., Althaus, D., Erhardt, J., Gloor, L., Hutter, A. \& Metzinger, T. (2015). Artificial Intelligence: Opportunities and Risks. Policy Paper by the Effective Altruism Foundation. Berlin: Effective Altruism Foundation, https://ea-stiftung.org / files / ai-opportunities-and-risks.pdf

Mosquera, J. (2015). «The Harm They Inflict when Values Conflict: Why Diversity Does Not Matter». Relations: Beyond Anthropocentrism, 3.

Næss, A. \& Sessions, G. (1999). The Deep Ecology Platform. En Witoszek, N. \& Brennan, A. (eds.) Philosophical Dialogues: Arne Nrss and the Progress of Ecophilosophy. Rowman and Littlefield, Oxford.

Næss, A. (1989). Ecology, Community and Lifestyle, Cambridge: Cambridge University Press.

Norton, B. (1987). Why Preserve Natural Variety? Princeton: Princeton University Press.

Nussbaum, M. C. (2006). Las fronteras de la justicia: consideraciones sobre la exclusión. Barcelona: Paidós, cap. 6 [título original: Frontiers of Justice: Disability, Nationality, Species Membership. Cambridge: Harvard University Press]

Pluhar, E. B. (1995). Beyond Prejudice: The Moral Significance of Human and Nonhuman Animals. Durham: Duke University Press. 
Plumwood, V. (1993). Feminism and the Mastery of Nature. New York: Routledge.

Ryder, R. D. (2010 [1970]). Speciesism Again: The Original Leaflet. Critical Society, 2.

Rachels, J. (1990). Created from Animals: The Moral Implications of Darwinism. Oxford: Oxford University Press.

Rolston III, H. (1999). Respect for Life: Counting what Singer Finds of No Account. En Jamieson, D. (ed.). Singer and His Critics. Oxford: Blackwell.

Rolston III, H. (1992). Disvalues in Nature. The Monist, 75.

Sagoff, M. (1984). Animal Liberation and Environmental Ethics: Bad Marriage, Quick Divorce. Osgoode Hall Law Journal, 22.

Shelton, J.-A. (2004). Killing Animals that Don't Fit In: Moral Dimensions of Habitat Restoration. Between the Species, 13/4, 1-21, http:/ / digitalcommons.calpoly.edu/bts / vol13 / iss $4 / 3$

Shiva, V. (1992) Staying Alive: Women, Ecology and Development. Atlantic Highlands: Zed Books.

Singer, P. (1979). Not for Humans Only: The Place of Nonhumans in Environmental Issues. En Goodpaster, K. \& Sayre, K. (eds.). Ethics and Problems of the $21^{\text {st }}$ Century. Notre Dame: University of Notre Dame Press.

Schweitzer, A. (1962 [1923]). Civilización y ética. Buenos Aires: Sur [título original: Kultur und Ethik, Bern: Paul Haupt]. Taylor, P. (1986). Respect for Nature, Princeton: Princeton University Press.

Tomasik, B. (2016 [2013]). «Which computations do I care about?», Essays on Reducing Suffering, http:// reducingsuffering.org/which-computations-do-i-care-about [visitado el 11 de enero de 2017]. 
(2010 [2009]). La importancia del sufrimiento de los animales salvajes. Essays on Reducing Suffering, http:// reducing-suffering.org/wp-content / uploads/2014/10/suffering-nature-Spanish.pdf [visitado en 12 de diciembre de 2016, título original «The Importance of Wild-Animal Suffering», versión revisada en 2015 en Relations: Beyond Anthropocentrism, 3, 133152, visitado en 12 de diciembre de 2016].

Torres Aldave, M. (2015). El fracaso de los argumentos contra la intervención en la naturaleza. En VV. AA, Los retos de la Filosofía en el siglo XXI: Actas del I Congreso Internacional de la Red Española de Filosofía, 17, Valencia: Universitat de València.

Varner, G. (2002). Biocentric Individualism. En Schmidtz, D. \& Willot, E. (eds.) Environmental Ethics: What Really Matters, What Really Works. Oxford: Oxford University Press.

Varner, G. (1991). No Holism Without Pluralism. Environmental Ethics, 13.

Warren, K. (2000). Ecofeminist Philosophy: A Western Perspective on What It Is and Why It Matters. Lanham: Rowman \& Littlefield.

Warren, M. A. (2000). Moral Status: Obligations to Persons and other Livings Things. Oxford: Oxford University Press.

Wenz, P. (1988). Environmental Justice. Albano: SUNY Press. Wobeser, G. A. (2005). Essentials of disease in wild animals. New York: John Wiley and Sons.

Zimmerman, D. (2009). Starvation and Malnutrition in Wildlife. Indiana Wildlife Disease News, 4. 


\section{Resumen}

Mientras las posiciones favorables a la consideración moral de los animales defienden que el criterio para ser objeto de respeto radica en la sintiencia, el ecologismo defiende otros criterios para proteger la consideración de entidades como los ecosistemas o especies. Esta diferencia acerca del criterio para la consideración moral tiene consecuencias prácticas muy importantes. Este artículo expone, en primer lugar, los principios en los que descansan el biocentrismo y el ecocentrismo, las objeciones que se pueden plantear y el modo en el que las diferentes posiciones en ética ambiental optan por posicionamientos antropocéntricos, que resultan cuestionables. Tras lo anterior, se expondrán los conflictos prácticos dados entre la consideración de los animales y el ecologismo, en particular en el caso de la intervención en el mundo natural para ayudar a los animales sintientes, y se explicará que se abre un conflicto entre el biocentrismo y el ecocentrismo.

Palabras clave: antropocentrismo, biocentrismo, ecocentrismo, ecologismo, ética animal, intervención.

\section{Abstract}

Biocentrism is the position that considers that morally considerable entities are living beings. However, one of the objections is that if we understand that moral consideration should depend on what is valuable, we would have to conclude that the mere fact of being alive is not what makes someone morally considerable. Ecocentrism is the position that holds that morally valuable entities are not individuals, but the ecosystems in which they live. In fact, among the posi- 
tions traditionally defended as ecologists there are serious divergences. Some of them, biocentrists, in particular, can ally themselves with holistic positions such as ecocentrism on certain occasions.

Key words: anthropocentrism, biocentrism, ecocentrism, environmentalism, animal ethics, intervention. 